Public health Association, New-York (éditions antérieures à la sixième).

[42] C. N. Stark et C. W. England (1935). Journ. Bact., 29, 26.

[43] - et L. R. Curtis (1936). Journ. Bact., 32, 375.

[44] Voges Proskauer (1898). Zeitschr. Hyg., 28, 20.

[45] G. S. WILSON (1935). "The bacteriological grading of milk», p. 153.

[46] - (1935). "The bacteriological grading of milk", p. 174.

[47] - (1935). "The Bacteriological grading of milk ", p. 181.

[48] - (1935). "The Bacteriological grading of milk ", p. 183.

[49] M. W. YALE (1933). Journ. Dairy Science, 16, 481.

[50] V. Zavagir (1933). Z. Infektkr., Haustière, 45 110.

\title{
LE LAIT DANS QUELQUES-UNES DE SES FORIMES D'UTILISATION
}

\author{
par le Dr José MaRia ROSELL
}

Bactériologiste du Ministère de l'Agrieulture de Québec.

Ancien professeur de Pathologie digestive et de la Nutrition,

à Barcelone (Espagne).

\section{INTRODUCTION}

Il paraîtra paradoxal et, pour le moins exagéré, de vouloir affirmer, comme nous le faisons ici, que le lait, le produit alimentaire, peut-être le premier qu'ait utilisé et connu l'homme, et en tout cas le premier que l'homme et les mammifères doivent utiliser pour former l'organisme après la naissance, et l'aliment sur lequel tant de louanges ont été répandues par les médecins et les profanes de toutes les époques, est un aliment, dont la raison de sa profonde importance pour la nutrition commence à être bien comprise et bien connue, seulement depuis l'époque moderne de la médecine et de la science de la nutrition et plus spécialement encore au cours des six ou sept dernières années.

En suivant les traités volumineux sur l'alimentation et la diététique, qui ont été publiés au cours des âges de la médecine, faisant référence en de longs chapitres à l'importance du lait pour l'alimentation de l'homme en état de santé et de maladie, et en feuilletant parmi les centaines de publications spéciales étudiant la valeur du lait et des produits dérivés du lait comme agent curatif ou thérapeutique, on pourrait être porté à croire qu'on ne peut plus découvrir de nouveaux faits scientifiques, montrant davantage, chaque année, la valeur du lait comme aliment et comme médicament.

Dans une des nombreuses publications des dernières années 
étudiant la valeur thérapeutique du lait, l'ouvrage du Professeur $\mathrm{D}^{\mathrm{r}}$ Rich. WeHsarg, intitulé "Modern Milchtherapie" (Thérapeutique lactologique moderne), cet investigateur connu, Conseiller de santé de l'Allemagne, nous dit, par exemple : "En étudiant ce que les médecins de l'antiquité, tant de la civilisation assyrienne que chaldéenne, égyptienne, indoaryenne, arabe, grecque et romaine, et d'autres civilisations primitives, ont écrit sur le lait comme aliment et comme médicament, on croirait se trouver devant des trésors médicaux, que les générations anciennes nous ont légués pour nous faire apprécier la valeur du lait et spécialement de certaines formes de lait, surtout des laits fermentés, tant pour maintenir et augmenter la santé que pour la recouvrer. "

Mais ce même auteur, en quelques autres endroits de son intéressant ouvrage, nous dit que si les sommités médicales des anciennes civilisations avaient déjà fait des apologies sur la valeur du lait, qui représentaient une formidable expérience des générations et des âges humains d'existence éloignée, et que si les savants des siècles postérieurs, physiologistes, pathologistes, médecins praticiens, officiers de santé et directeurs d'Etat, ont continué cette œuvre pour étudier et nous faire eonnaître la valeur du lait, il manque encore bien de longues études et recherches pour que nous arrivions à trouver la cause biologique finale qui soit capable de nous expliquer pourquoi ce produit admirable, appelé le sang blanc ou l'organisme vivant en forme de liquide, possède des pouvoirs si grands pour la nutrition et la santé.

Si l'on fouillait les centaines ou plutôt les milliers de publications de la bibliographie relative au lait, on pourrait trouver de nombreuses attestations pareilles à celle que nous venons de reproduire en exemple. A la fin de ce court opuscule dédié à l'étude de quelques-uns des aspects ou formes de présentation du lait, au point de vue de ses avantages diététiques et autres, nous donnons le titre de quelques références faciles à être consultées en faisant référence aussi à quelques publications sur le lait étudié au point de vue digestif et médical que nous avons eu occasion de publier nous-même, après des travaux cliniques et de laboratoires que nous avons faits il y a quelques années. Ceux qui seront intéressés pourront, en ces publications mentionnées, puiser de plus amples informations sur la question de lactologie moderne et ancienne, que celles que nous pouvons fournir en cet article qui veut seulement présenter une révision sommaire de quelques-unes des formes d'utilisation, autres que l'ordinaire, et faire ressortir l'importance de cet aliment "le plus parfait de tous les aliments connus ". 


\section{PREMIËRE PARTIE}

\section{LE LAIT «ALIMENT »}

De tous les produits alimentaires, le lait, première nourriture que l'homme et tout animal mammifère trouve préparée à sa naissance, est, sans doute, répétons-le, le plus important. Quelques physiologistes l'appellent le nourrisson en forme liquide, parce que les cendres du lait ont exactement la même composition que les cendres du nouveau-né. Mais, quel que soit le nom que l'on adopte ou les louanges qu'on lui donne, e'est un fait indiscutable que, pour chaque espèce de nourrisson ou d'être vivant qui tette, le lait maternel est la seule nourriture physiologique rationnelle. C'est le seul aliment, pour le jeune organisme à cet âge, pouvant être appelé " complet». Le lait contient exactement tout ce dont l'enfant nouveau-né a besoin pour son développement, jusqu'au moins le septième ou le neuvième mois. D'autres auteurs ont encore appelé le lait une "viande liquide", mais improprement, parce que le lait, en soi, est un aliment plus complet que la viande.

Pour l'homme adulte lui-même, de même que le pain complet lequel est presqu'inconnu des générations modernes de notre pays -, le lait sera toujours l'aliment le plus complet bien que pas toujours suffisant.

Le lait, produit des cellules de la glande mammaire, possède nombre de caractères biologiques du sang et pour cela on l'appelle parfois le sang blanc. Le lait est destiné par la nature à se substituer au sang à la naissance des nouveau-nés. Il remplace le sang de la mère au moment où le fotus sort de la cavité utérine. De même que plusieurs de ses dérivés, en plus de ses qualités enzymatiques et vitaminiques, il possède d'autres qualités vitales telles, par exemple, les qualités immunisatrices et même bactéricides, celles-ci s'opposant, dans un certain degré, au développement de bactéries nuisibles à l'organisme, et favorisant le développement des bactéries les plus utiles chez l'homme : les bactéries lactiques.

Le lait est considéré, on le sait, comme l'unité de mesure de la nutrition, comme le mètre est l'unité de mesure des distances. Von Pirquet, une des plus grandes autorités en alimentation, en exposant la thèse que le lait est indiscutablement la nourriture la plus parfaite, créa le « Nem » ou Unité alimentaire du lait (Naehrunge Einheit Milch), qu'on utilise aujourd'hui fréquemment au même titre que la mesure "les calories".

Le lait est une merveille physiologique; il contient toutes les substances nécessaires pour la vie: protéines, l'pides, glycides, sels, vitamines, etc., et dans les proportions nécessaires pour l'organisme humain, surtout dans le jeune âge, et aussi dans une 
forme bien adaptée à l'appareil digestif de l'enfant. Il n'était que très raisonnable et désirable qu'on en tînt compte, au moyen du "Nem ", dans l'évaluation des nécessités alimentaires, non seulement en calories, mais aussi de toutes les nécessités nutritives (caloriques, plastiques, minérales et même vitaminiques).

De plus le lait est considéré comme la source de protéines alimentaires la plus économique et la plus complète en acides aminés que l'on puisse off ir à l'organisme humain ou animal.

Dans tous les pays, les médecins, les hygiénistes et les dirigeants s'efforcent de convaincre chaque jour les populations que la santé et la vigueur des générations présentes et futures sont intimement liées à une abondante consommation des produits laitiers.

Nous avons dit, en nous basant sur l'enseignement médical à travers les siècles, que les ouvrages des médecins, depuis ceux de l'antiquité (Galìne, Hippocrate, Avicennes, etc.) jusqu'aux plus modernes, sont remplis des louanges du lait et particulièrement sous les diverses formes des produits dérivés, tels que les laits fermentés : le Yoghourt, le Kéfir, etc, utilisés dans les temps anciens comme médicament et comme nourriture hygiénique de la plus haute valeur nutritive. Le lait est donc considéré, surtout par ceux qui se sont livrés à de longues recherches sur son utilisation, comme un des éléments de cure médicale les plus importants pour un grand nombre de maladies et comme aliment de première qualité pour toutes les phases de la vie.

Ce n'est done pas sans raison que les gouvernements et les membres des bureaux d'hygiène de plusieurs pays apprécient chaque jour davantage la haute valeur du lait et de ses dérivés, beurre et fromage, pour la santé individuelle et l'avenir de la race, et se préoccupent de plus en plus de faire connaître cette importance, par une active propagande. Un des efforts les plus remarquables de l'actualité, parmi les directeurs ou responsables de la santé en de nombreux pays, c'est la substitution, à toutes ces boissons douces du commerce : limonades à la mode, limonades à essence, toutes plus ou moins artificielles et pour la plupart de presque nulle valeur nutritive ou hygiénique et souvent même toxiques par leur contenu en substances préservatrices nuisibles à la santé, des boissons rafraîchissantes, nutritives et hygiéniques à base de lait fermenté, ou de lait ou sérum additionnés de jus de fruits.

Les champagnes de lait ou laits mousseux, tels que faits actuellement et qui jouissent d'une si grande vogue dans les pays où ils ont été introduits, constituent un grand progrès dans l'amélioration hygiénique des breuvages et surtout dans les possibilités de présenter le lait sous des formes avantageuses, à la portée de nom- 
breuses personnes qui ne peuvent pas ingérer le lait en sa forme naturelle, soit par répugnance ou manque d'attrait ou le plus fréquemment par intolérance digestive ou d'autre sorte.

Le jour où la consommation trop abondante de boissons douces. sera remplacée par des boissons rafraîchissantes à base de lait incomparablement plus avantageuses, tant au point de vue de la santé que de l'économie : les exquis champagnes au lait très en vogue, les Yoghourt, lait de beurre de culture, lait à l'orange et autres boissons de jus de fruits et lait ou sérum de lait... ce jour-là, la force, la santé, même la beauté et autres aspects de la population seront améliorés considérablement.

\section{Courte revision préliminaire de quelques très importantes et récentes acquisitions de la science de la nutrition et du métabolisme}

Je crois qu'il y a intérêt, pour ceux qui n'ont pas toujours l'occasion de suivre la bibliographie des différents pays sur ces matières, de présenter un résumé des quelques conclusions et concepts actuels sur le lait "aliment» d'une valeur toute spéciale dans la diététique moderne et dans la médecine, comme aussi de jeter un regard rapide sur les facteurs que la physiologie moderne de la nutrition considère comme étant les plus importants pour interpréter le rôle décisif que le lait joue réellement.

La recherche scientifique en biologie et médecine des dernières années a montré, et cela comme une remarquable caractéristique, une plus grande attention aux différents facteurs isolés de l'alimentation, qui ont une influence décisive sur la santé en général, sur la résistance ou prédisposition aux maladies, et sur l'amélioration de l'appauvrissement ou déficience de la constitution de l'individu, et, par là, de la race. Ces études sur l'influence des différents facteurs de l'alimentation dans la santé ont été poursuivies et étudiées largement, tant dans la race humaine, que dans les races animales, spécialement celles intéressant la zootechnie agricole.

Il ne serait pas actuellement possible à une seule personne de suivre toutes les publications qui, dans les vingt ou vingt-cinq dernières années, ont paru dans les différents pays sur l'alimentation en relation avec la santé et la maladie. On ne pourrait même pas recueillir une information complète sur les travaux faits en certains domaines isolés de la science de nutrition, par exemple sur tous ceux qui se rattachent à la connaissance du métabolisme minéral, des vitamines ou autres facteurs fondamentaux.

Les points que je veux exposer ici, dans la forme la plus résumée possible, sont ceux qui se rapportent aux défauts de l'alimentation de la population des centres trop industrialisés, alimentation qui, 
comme l'ont fait remarquer à plusieurs reprises d'éminents physiologiste 3 et hygiénistes, présente des défauts bien funestes pour l'individu, la population et la race même. Une des déficiences nutritives principales, pour la plus grande partie des populations civilisées, consiste en une insuffisance de certains types de protéines et de certains minéraux, entre autres le calcium, un des plus nécessaires pour l'organisme.

La qualité des protéines est d'une grande importance pour une alimentation correcte.

La quantité de protéides ou matières azotées compte dans la ration; mais le type ou qualité de protéine est de la plus grande importance. Ce fait, trop mal connu par la physiologie et la pathologie, a pris, depuis quelques années, une nouvelle signification et une plus grande importance, à la suite des recherches plus approfondies du métabolisme qui ont été faites surtout à l'Institut de Physiopathologie de Berlin pendant les cinq ou six dernières années.

Ayant pu suivre de près - et récemment sur place - ces études, et ayant pu aussi obtenir l'évidence de sa grande importance, spécialement au regard de quelques aspects de l'alimentation de la population canadienne, nous croyons que la possibilité d'avoir quelques renseignements sur cette question vitale peut constituer un progrès d'ordre général, même si, pour pouvoir les exposer en prenant une base physiologique, nous sommes obligés de considérer en passant quelques points du métabolisme ou des échanges nutritifs.

La recherche physiologique et l'expérience médicale avaient établi depuis plus d'une quarantaine d'années, que la quantité de substances azotées qui devaient être consommées par l'homme adulte en conditions ordinaires de santé et de travail, étaient comprises entre 72 grammes minimum de protéines par jour par individu et 110 au maximum. Une consommation en matières proté niques plus grande que 110 grammes ou plus petite que 72 grammes est considérée par la physiologie actuelle - et cela avec raisons bien fondées - comme incompatible à la longue, avec la santé (96 grammes était le taux souvent donné comme un chiffre moyen recommandable).

On avait établi les proportions de matières azotées, fécules, et corps gras de tous les aliments connus, lesquelles proportions, présentées dans les tableaux connus, complétées par les valeurs en vitamines, matières minérales, valeurs caloriques, etc., servent de base pour l'établis ement des régimes ou menus raisonnés.

En des périodes plus récentes de l'avancement de la science de la nutrition, tant dans son application humaine qu'animale, on 
reconnut bientôt que les albumines des différents aliments animaux ou végétaux ou protéines, n'étaient pas d'une égale importance quant à leurs effets sur la croissance et la réparation des pertes de tous les organes et tissus. On reconnut bientôt que ni l'albumine ou protéine de la viande, surtout la substance musculaire, ni, non plus, les albumines des œufs et surtout les albumines végétales, utilisées sous une forme exclusive, ne pouvaient suffire à satisfaire toutes les nécessités de l'organisme, et principalement, des organismes en développement ou croissance. Des aliments naturels, même de composition apparemment complète d'après leurs valeurs caloriques et même vitaminiques et minérales, étaient insuffisants au point de vue vital. Le lait, seulement (et les dérivés du lait, tels les fromages, contenant toutes les substances essentielles du lait), pouvait faire exception et pouvait être considéré comme le seul aliment complet, suffisant pour tous les besoins vitaux de la première période de croissance et presque complet pour les périodes ultérieures.

Depuis des années, on attribuait ce privilège spécial pour la construction et l'entretien de l'organisme, surtout à la bonne proportion des substances qui se trouvent dans le lait, tels que les protéines, hydrocarbones, matière grasse, minéraux et vitamines, et à sa facile digestion. Mais, plus tard, on fut capable de démontrer que la place toute spéciale qu'a le lait comme aliment unique, provient principalement de la qualité de ses protéines, les seules qui contiennent tous les acides aminés dont ont besoin, pour s'édifier, se maintenir et se renouveler, les protoplasmes ou corps protéiniques spécifiques des différents organes : viscères, muscles, glandes, etc. L'expérience ancienne, comme aussi la science de l'alimentation, montraient que le lait et ses dérivés (spécialement parmi ceux-là, les fromages de facile digestion) paraissaient être les nourritures les plus complètes et convenables pour l'organisme, à différents points de vue.

Mais une démonstration scientifique dans l'établissement des formules alimentaires tant pour l'homme que pour les animaux, manquait jusqu'à ce jour, et cette démonstration a été réalisée à l'institut de Physiologie pathologique que nous avons nommé plus haut, par les travaux du Professeur Dr Adolf SickeL, l'une des plus puissantes autorités mondiales dans la science de la nutrition et premier Directeur de l'Institut de Physio-Pathologie de l'Université de Berlin, travaux qui ont ouvert de bien importants et nouveaux horizons à la science de l'alimentation.

De ces remarquables constatations que nous allons résumer, on pourra, sans autres explications, déduire les applications que l'on fait et que l'on peut faire en vue d'une plus parfaite et salutaire 
alimentation, en même temps que l'on y trouvera une nouvelle confirmation et explication des avantages qu'il y a à utiliser le plus grand pourcentage possible de protéines de lait comme protéines alimentaires ordinaires.

On sait, surtout depuis HopkINGs, que les protéines ingérées jouent un rôle différent dans les fonctions constructives et énergétiques selon leur quantité, et l'on sait qu'il y a des protéines de différentes valeurs biologiques. On savait aussi qu'il existe une influence précise de la quantité des protéines ingérées sur les étapes d'oxydation de tout le processus du métabolisme anabolique et catabolique et on reconnaissait déjà qu'on pouvait obtenir un effet potentiel en combinant dans la ration, des protéines riches en différents acides aminés. De même, on avait établi qu'une alimentation sans protéines, bien que composée d'un mélange calorique suffisant, était suivie d'une forte diminution des oxydations et du métabolisme basal. Mais il restait encore de nombreuses lacunes dont certaines ont commencé d'être comblées par les travaux spéciaux auxquels nous faisons allusion.

Les résultats des recherches de BICKEL et de ses collaborateurs sont obtenus, en grande partie, par l'utilisation des nouvelles méthodes d'étude des phénomènes intimes du métabolisme que nous avons pu voir appliquer mais que nous ne pouvons décrire tout au long ici. Nous en extrayons l'essentiel :

(1) Les matières protéiniques interviennent d'une manière fondamentale dans les deux formes du métabolisme, autant dans le métabolisme constructif que dans le métabolisme fonctionnel. Cet effet, appelé "dynamique spécifique des protéines", dépend des acides aminés et différe pour chaque protéine. Il dépend de la quantité et de la qualité des acides aminés ou pierres fondamentales de la construction de l'édifice de chaque protéine. Ces acides aminés ont une action stimulante et spécifique sur les processus d'oxydation dans le métabolisme, facteur qui décide la capacité spécifico-dynamique ou potentiel énergétique de chaque type de protéine.

2. Cette capacité d'effets spécifiques dynamiques des protéines ou matières azotées ne dépend pas seulement de la quantité, mais, comme il a pu être démontré avec les nouvelles recherches, dépend aussi essentiellement de la qualité ou type de la protéine. L'effet ou potentialité spécifico-dynamique de chaque protéine est le résultat ou somme des valeurs spécifiques dynamiques de chacun des acides aminés qui entrent dans la construction de l'édifice de la molécule protéique, molécule qui, comme on le sait, présente des architectures moléculaires les plus variées avec des poids moléculaires énormes. Le poids moléculaire de la caséine, par exemple, 
peut être évalué à environ 16,000 . Le mécanisme de cette action spécifico-dynamique dépend, en grande partie, des processus de désamidation et du travail spécifique des protoplasmes de chaque cellule au cours du stade intracellulaire du catabolisme : il est nécessaire pour le travail énergétique et plastique ou anabolique des synthétisations de chaque cellule des différents tissus de l'organisme. Un même type d'aliment, hydrocarboné et gras, est utilisé ou oxydé différemment par l'organisme, selon la qualité des matières azotées qui l'accompagnent ou qui font partie du type d'alimentation ou d'épreuve choisie. Ces régimes d'épreuves standardisés se composent d'aliments exactement balancés et mesurés principalement en leurs proportions hydrocarbonées, grasses, minérales, et vitaminiques, et aussi en leur quantité d'azote ou protéine. Le seul facteur qu'on fait varier est le type de protéine.

3. D'autres résultats également importants de ces recherches ont été la démonstration :

a) Que tous les acides aminés n'ont pas les mêmes pouvoirs énerg tiques, nutritifs ou spécifico-dynamiques.

b) Que cet effet peut être multiplié par l'association de certaines protéines selon leur contenu en acides aminés spécifico-dynamiques.

c) Qu'il y a aussi des acides aminés apparemment dépourvus des actions spécifico-dynamiques.

4. La présence d'un minimum d'acides aminés spécificodynamiques dans l'alimentation est indispensable pour maintenir le niveau d'oxydation nécessaire pour une bonne nutrition et un bon état de santé. Cette présence est nécessaire pour une correcte utilisation des autres éléments de la ration ingérée, comme elle est également nécessaire pour un échange nutritif normal et optima. La présence d'acides aminés en quantité et qualité déterminées, est une condition essentielle.

5. Les acides aminés ou pierres fondamentales pour la construction et l'entretien de l'organisme ne sont pas tous contenus dans toutes les protéines ou tous les aliments azotés. D'une longue étude comparative faite sur cette question (dans l'index bibliographique à la fin de cet article nous mentionnons 18 thèses expérimentales sur l'échange du métabolisme, publiées sur ce sujet dans les six dernières années), étudiant la valeur nutritive et spécificodynamique comparative des différentes protéines animales et végétales, on a montré des variations considérables dans la valeur nutritive des différents aliments à ce sujet : ainsi la protéine la plus parfaite, puissante et complète au point de vue nutritif pour l'enfant et l'adulte, c'est la caséine du lait.

Cette protéine du lait se révèle, d'après un grand nombre de 
recherches expérimentales récentes, considérablement supérieure au point de vue nutritif et au point de vue des actions spécifico-dynamiques et énergétiques pour l'organisme, à toutes les protéines connues. Les protéines du lait, la caséine surtout, mais, plus spécialement, la caséine et la lactalbumine combinées, se sont montrées, au cours des recherches effectuées ces dernières années, considérablement supérieures aux protéines de tous les autres aliments : la viande, les œufs, les poissons, etc., et encore davantage aux protéines végétales qui sont celles ayant le plus bas effet spécifico-dynamique et les moins bien utilisées par l'organisme.

De ces résultats, et de beaucoup d'autres, dont nous ne pouvons pas faire mention ici, on est arrivé à la conclusion générale que la qualité de la protéine a une importance et un effet beaucoup plus profond sur la nutrition que tout ce qu'on avait supposé et reconnu jusqu'à cette date. Mais la conclusion la plus importante a été la démonstration de l'importance du lait comme base fondamentale de l'alimentation surtout comme source protéinique tant dans le jeune âge que dans l'âge adulte.

Dans le tableau schématique que nous donnons ci-après, sur la composition chimique du lait, tableau qui réunit à peu près toutes les connaissances actuellement certaines sur la composition du lait, on pourra trouver la liste des acides aminés qui contribuent à l'architecture de la molécule de caséine, et aussi des autres éléments fondamentaux de nutrition (matières protéiques, matières grasses, glycides, matières minérales, vitamines et autres substances accessoires et de défense). Le lait apparaît ainsi constituer non seulement une substance alimentaire dans le sens ordinaire du mot, mais une source d'énergie et de facteurs vitaux qui garantissent en même temps que la nutrition, la santé et la résistance de l'homme vis-à-vis de presque toutes les maladies et, en même temps, comme la clinique expérimentale de toutes les époques l'a démontré, une source de santé et on peut dire sans embarras, une médecine de tout premier ordre contre de nombreuses maladies, surtout si on utilise le lait à la fois sous sa forme naturelle, et sous forme de ses nombreux dérivés, dont certains sont des médicaments, et que nous voulons, ici, passer en revue.

\section{COMPOSITION MOYENNE DU LAIT DE VACHE}

Lait $100 \mathrm{~cm}^{3}$

Eau ....... 87,55

Matières sèches

12,45
Matière sèche

Matière grasse . . 3,70

Mat, sèche non

100,00 grasse .......
8,75

12,45
Matière sèche non grasse

Matières zotées .. 3,25

Sucre de lait et acide citrique . . 4,80 Matières minérales 0,70 
Gaz :

$\begin{array}{ll}\mathrm{CO}^{2} & 6 \% \\ \mathrm{~N}^{2} & 3 \% \\ \mathrm{O}^{2} & 1 \%\end{array}$

Matières azotées

Caséine ......... 2,50

Lactoalbumine ..... 0,50

Albuminoses ........

Caséoses .........

Nucléone .........

Opaleseine.........

Lactochrome ......

Fibrine, etc. .......

Lécithines et stérols .
Globuline ........

Acides aminés de la caséine et lactoalbumine

Caséine Lactoalbumine

Alanine $\ldots \ldots \ldots \ldots \quad 0,9 \quad 2,5$

Valine ......... $1,0 \quad 0,9$

Leuzine ....... 10,5 19,4

0,20 Phenylalanine .... $3,2 \quad 2,4$

Tyrosine $\ldots \ldots \ldots \ldots \quad 4,5 \quad 0,9$

Serine ....... $0,3 \quad$

Cystine ........ 0,1 -

0,05 Proline ........ $3,1 \quad 4,0$

- Oxyoroline...... - - 0,3

3,25 Acide asparaguinique $1,2 \quad 1,0$

Acide glutaminique. $\quad 10,7 \quad 10,1$

Tryptophane ..... 1,5 -

Arginine $\ldots \ldots \ldots \ldots$ 4,8 -

Lysine $\ldots \ldots \ldots \ldots \quad 5,6 \quad-$

Histidine ...... 2,6

Matière grasse du lait

Point de fusion : commence à $19^{\circ}$ finit à $43^{\circ}$.

Point de solidification : commence à $21^{\circ}$ finit à $16^{\circ}$.

Indice d'iode $30^{\circ}-40^{\circ}$.

Indice de saponification 216-236.

Indice Polenske 2,1-2,6.

Indice Reichert-Meissl 25-27.

Acides gras non volatils de la matière

Acides gras volatils grasse

\begin{tabular}{|c|c|c|c|c|}
\hline Oléine .. & $33,95 \%$ & & Butyrine & $6,23 \%$ \\
\hline Palmitine & $40,51 \%$ & & Caproïne & $2,32 \%$ \\
\hline téarine. & $2,95 \%$ & 3,40 & Capryline & $0,53 \%$ \\
\hline Iyristine & $10,44 \%$ & & Caprinine & $0,34 \%$ \\
\hline
\end{tabular}


Haptènes et antigènes ou corps

Enzymes

immunisants du Jait

Réagine...........

Baetériolysine .......

Cytolysine $\ldots \ldots \ldots$.

B. Diverses $\ldots .\left\{\begin{array}{l}\text { Oxydases } \\ \text { Réductases } \\ \text { Catalases }\end{array}\right.$

Quelques caractères physiques de lait

Poids spécifique : $1.033-1.032$ à $150^{\circ} \mathrm{C}$.

Point de congélatió : 0,55 .

Viscosité : $2,00-2,20$ à $15^{\circ} \mathrm{C}$.

Valeur calorique ou de combustible : 670 .

$p \mathrm{H}: 6,4-6,6$.

\section{Quelques conclusions finales sur le lait « aliment »}

Les principaux facteurs qui décident de la valeur digestivenutritive, hygiénique et même médicale du lait sont les suivants :

A. Les protéines du lait sont, telles qu'énoncées plus haut, les plus complètes de toutes les protéines connues (animales ou végétales). Elles contiennent tous les acides aminés (voir le tableau) ou les pierres angulaires de tout l'édifice organique de l'homme et de l'animal, c'est-à-dire, tous les groupes ou constellations moléculaires qui permettent la formation synthétique par l'organisme, des protéines spécifiques de chaque tissu ou organe. Les meilleures protéines animales et végétales sont, contrairement à la caséine, toujours déficientes en quelques-uns des acides aminés de croissance ou d'entretien, par exemple, lysine, thyrosine, tryptophane et autres acides aminés absolument indispensables pour l'édification et la construction des différents organes.

Pour suppléer aux déficiences spécifiques des protéines composant la ration alimentaire ordinaire des populations des grandes et souvent aussi des petites villes, il faudrait ajouter à celle-ci, une quantité d'environ un litre de lait. Dans un régime diététique à base presque exclusive de lait, il faudrait apporter de 21.500 à 31.500 de lait par jour par personne, ou quantités équivalentes de fromage bien digestible (par exemple : fromage frais à la crème ou fromage du type Bel-Paese, Port-Salut, Richelieu, Gouda et autres fromages mous à maturation lactique complète). Les fromages de plus profonde maturation, possédant la plus grande digestibilité, sont les types "Bel Paese", Camembert, Brie, Roquefort entre autres.

Les protéines du lait, ingérées sous des formes convenables, sont plus digestibles que celles des autres substances alimentaires animales et végétales. Ceci est attribuable, surtout pour les laits fermentés et les fromages, à l'action des enzymes de la maturation qui produisent généralement une prédigestion de 30 à $95 \%$ des 
protéines et aux enzymes contenues par ces produits. Les protéines du fromage "Richelieu ", par exemple, sont digestibles, d'après les expériences médicales, jusqu'à $100 \%$ de sa substance.

B. Ingérées en bonnes conditions diététiques, les protéines du lait sont absorbées presque intégralement par l'intestin. Avec une alimentation carnée, les pertes de matériel azoté dans les excréments sont bien plus considérables, soit par défaut de digestion, soit par défaut d'absorption. Encore plus considérables sont les pertes produites par un régime de protéines animales autres, et de protéines végétales. De là, on considère le régime à base de lait et des dérivés du lait comme un régime antiputride.

Dans un travail sur la physiopathologie digestive du lait, conduit pendant plus de 2 années, et portant sur la détermination coprologique des substances protéiniques absorbées et utilisées par des individus sains ou affectés de maladies digestives différentes, nous avions fait en 1918-1919 une ample démonstration de ces faits énoncés en ce paragraphe (1), et d'autres travaux, publiés depuis, ont abouti à la même constatation.

C. Les protéines du lait sont absorbées et fixées par l'organisme dans une proportion plus forte que toute autre substance protéique; elles ont un pouvoir de contribution à l'oxydation et à l'utitisation des autres aliments, plus grand que toutes les autres protéines.

D. Les matières grasses du lait, par leur état d'émulsion et, dans quelques laits modifiés et divers fromages, souvent à la suite de dédoublement lypasique et par leur point de fusion de $93^{\circ} \mathrm{F}$. (température inférieure à celle de l'organisme), constituent la matière grasse la plus digestible et absorbable de tous les aliments gras. De plus, le mélange intime et presque colloïdal de la matière grasse avec les protéines et autres substances du lait, saturées pour ainsi dire, en certaines présentations de lait et produits laitiers, d'enzymes digestives (lipases) et de substances stimulantes, est un puissant facteur de la digestion facile des matières grasses du lait.

E. Le lait contient, en un mélange idéal, les matières minérales nécessaires à l'organisme, surtout les sels de chaux, lesquels sont toujours déficients, dans une proportion élevée, dans la nourriture ordinaire. Toutes les autorités de la science de la nutrition sont d'accord depuis longtemps pour reconnaître que la cause d'un grand nombre d'altérations bien connues de la santé ainsi que la prédisposition à des maladies infectieuses et inflammatoires (tendances

(1) J. M. Rostelt. Physiopathologie digestive du lait et les fermentations et putréfactions intestinales, Barcelona, Libreria Nacional y Eatranjera, 1920. - Arch. Pathology, Digest., Madrid, 1919, no 5-6. - Rosell, Coprologie Clinique, Vigot Paris, 1927. - Studies in the pathology of Digestion, Canada Med. Ass. Journal, 1929, no $30,42-46$ et $161-168$. 
spasmophiliques, hypotonie de la fibre musculaire lisse, etc, en plus des débâcles dentaires ordinaires et du manque de formation squelettique), sont surtout dues à une insuffisance calcique. On estime le besoin journalier en chaux, pour une personne adulte, à environ 1 gr. $25-1$ gr. 75 . Toute nourriture renfermant moins de cette quantité de chaux est insuffisante et, par conséquent, néfaste pour la santé. Sur ce point, on peut dire ce qu'affirment tous les physiologistes qui se sont occupés de la question : "que toute nourriture composée essentiellement de viande, de pommes de terre, de beurre ou matières grasses, de farine, de sucre, de pain blane est fortement déficiente en sels de chaux et autres sels." Ces régimes ne contiennent pas même 1 gramme de chaux; ils constituent une nourriture insuffisante et prédisposent à des troubles de santé. Ceci est un fait confirmé et indiscutable. Seulement, l'addition d'une quantité régulière de $1 / 2$ à 1 litre de lait par jour environ, ou l'équivalent en fromage ou en choux blancs (l'aliment végétal le plus riche en chaux), ou en pain de blé entier, peut corriger les conséquences néfastes d'un tel régime alimentaire; et, pourtant, ce régime déficient est le régime ordinaire de la grande partie de la population des pays civilisés.

Un litre de lait de vache contient 1 gr. 50 à 2 grammes de sels de chaux.

Un litre ou au moins un demi-Jitre de lait, ou trois onces (100 grammes) de fromage, par jour, ajoutés à l'alimentation ordinaire, constituent le meilleur remède recommandé instamment par les autorités médicales ( $J$. LoEB MACCALLUM et d'autres), pour corriger les troubles dus à la déficience de chaux dans l'alimentation ordinaire.

Le calcium dans le fromage et dans le lait se présente sous la forme du calcium le plus assimilable par l'organisme. Le calcium des sels minéraux, à l'exception du chlorure $\left(\mathrm{CaCl}^{2}\right)$ et lactate de chaux, est très peu, pour ne pas dire pas du tout, utilisable par l'organisme. Les phosphates et carbonates de chaux officinaux ne sont pas absorbés par l'intestin.

Nous ne pouvons parler ici de l'importance des autres substances minérales du lait qui contiennent, comme nous l'avons dit, tous les éléments minéraux utiles à l'organisme, et cela dans la proportion requise. Dans un ouvrage sur la physiologie et la pathologie du métabolisme minéral (1), j'ai exposé cette question avec maints détails.

F. Le lait, en général, a été appelé "l'aliment de protection 》.

(1) J. M. Roselt. Métabolisme minéral. L'importance physiologique, pathologique -et hygiénique des éléments minéraux contenus dans les aliments, Barcelone, 1927, 2 volumes. 
G. En raison du grand nombre d'enzymes des ferments lactiques et de ces ferments dans certaines variétés de laits modifiés comme aussi dans de nombreux fromages mous affinés, ces aliments aident la digestion des autres aliments ingérés. Même le fromage Cheddar bien "à point ", lorsque l'insalivation est bonne, constitue un produit de digestion facile. Dans bien des pays, en raison de leur effet digestif, on considère les laits fermentés et le fromage comme un complément indispensable du repas, en recherchant surtout ainsi les fromages bien affinés, tels que le Camembert, le Roquefort, le Brie, le Bel-Paese, etc.

H. Le lait est, par son lactose, une source d'acide lactique surtout abondant en certains dérivés du lait.

Les physiologistes modernes considèrent que l'acide lactique est une des substances les plus importantes pour presque toutes les fonctions de l'organisme.

Entre autres, l'influence de ce produit est très importante, pour l'accomplissement normal des fonctions digestives, museulaires, reproductrices, nerveuses, glandulaires et endocrines. De plus, il faut considérer le pouvoir antiputride de cet acide et son pouvoir défensif contre les infections et les intoxications non seulement alimentaires mais aussi d'origine métabolique. C'est cette influence sur les métabolismes intermédiaires qui a donné une base aux théories actuelles, assez bien confirmées, selon lesquelles l'acide lactique est la substance qui empêche la sénilité (vieillissement) précoce des tissus. On appelle l'acide lactique (voir Boris SoKoLOFF, "Vitality", 1936) le "suprême régulateur", le " combustible du cerveau " et le "pro-enzyme universel ".

Même au point de vue du mécanisme de l'immunité naturelle contre les infections, on a pu démontrer que l'acide lactique est une des principales co-hormones nécessaires à l'accomplissement de la phagoeytose. Sans acide lactique, les leucocytes sont incapables d'exercer leurs fonctions défensives.

Au cours des quatre dernières années un travail considérable s'est élaboré sur l'importance et la signifieation de l'acide lactique, et les résultats obtenus semblent nous révéler le début d'une ère nouvelle en biologie, en hygiène et en médecine. 